\title{
Technology Research and Performance Analysis of Bonded Piston Seal
}

\author{
XU Xiao-yang ${ }^{1, a^{*}}$, XU Xiang-yang ${ }^{2, b}$
}

${ }^{1}$ Beijing Key Laboratory for High-efficient Power Transmission and System Control of New Energy Resource Vehicle, School of Transportation Science and Engineering, Beihang University, 37 Xueyuan Road, Haidian District, Beijing 100191, China

${ }^{2}$ Beijing Key Laboratory for High-efficient Power Transmission and System Control of New Energy Resource Vehicle, School of Transportation Science and Engineering, Beihang University, 37 Xueyuan Road, Haidian District, Beijing 100191, China

axuxiaoyang325@163.com, bxy@buaa.edu.cn

Key words: Electric vehicle; Automatic transmission; Bonded Piston Seal; Rubber material; Ansys.

Abstract. BPS(Bonded Piston Seal) is currently widely used in automatic transmission. In terms of the BPS in an in-development two-speeds automatic transmission of an electric vehicle, this article makes a detailed introduction to BPS technology and carries out a comprehensive analysis. The first part is an introduction to BPS. After that, this article analyzes the working environment and the technical requirements of BPS. The second part is the study on rubber material characteristics of BPS. Then a design process of the BPS in a two-speeds automatic transmission of an electric vehicle is demonstrated. According to the design, Ansys is used to build models and analyze the pressure distribution of the upper lip and the lower lip of the BPS. Then the author analyzes the sealing performance and makes structural improvement. Finally reasonable structural drawings are obtained.

\section{Introduction}

Bonded Piston Seal. Piston Seal is an important part of the design of automatic transmission. It includes O type, X type, long/short lip type, BPS and so on.

The structure feature of BPS is that there are one or more lips in the ring section, and BPS works with the lips closely against the inner wall of the cylinder[1]. BPS is usually made of rubber material and metal skeleton after vulcanization and the second vulcanization. Some figures of BPS are as follows, see Fig. 1.
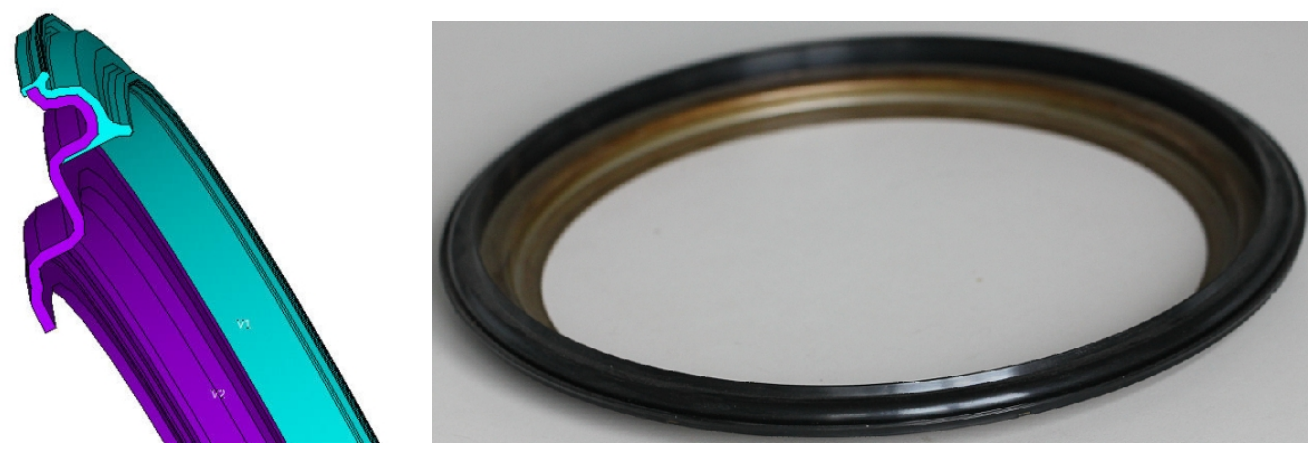

Fig. 1 Bonded Piston Seal

Bonded Piston Seal has many advantages. For example, it has advantages of small compression deformation, automatic compensation to the wear of BPS, little leak quantity, better sealing performance, , high reliability and a long service life. Moreover, BPS is an integral unit, unlike other units which consist of three to five parts. Thus it has been widely used in automatic transmission in electric vehicle.

Technical requirements. Since the BPS in automatic transmission works in a complex and demanding environment, and it requires a long service life, the technical requirements of BPS are very harsh. Technical requirements are shown below[2]: 
(1) High distortion resistances and high hardness;

(2) High resistance to ATF;

(3) Resistance to high and low temperature: $-40^{\circ} \mathrm{C} \sim 150^{\circ} \mathrm{C}$;

(4) Shear strength of $6 \mathrm{MPa}$;

\section{Material analysis}

Rubber material analysis. Rubber material is the main factor for the sealing performance and the service life of BPS. Three kinds of commonly used rubber material are HNBR, AEM, FKM. Each of them have advantages and disadvantages. The performance analysis of each of them are shown in Table 1.

Table 1 Performance comparisons of HNBR, AEM, FKM

\begin{tabular}{cccc}
\hline Performance & HNBR & FKM & AEM \\
\hline Density[g/cm ${ }^{2}$ ] & 1.24 & 1.85 & 1.10 \\
Tensile strength[MPa] & 25 & 13 & 16.3 \\
Hardness & 81 & 80 & 84 \\
Compression resilience ratio[\%] & 28 & 7 & 30 \\
Tearing strength[MPa] & 19 & 16 & 12 \\
Distortion resistances $\left(150^{\circ} \mathrm{C} \times 70 \mathrm{~h}\right)[\%]$ & 23 & 13 & 15.07 \\
Max temperature $\left[{ }^{\circ} \mathrm{C}\right]$ & 170 & 315 & 180 \\
Brittleness temperature $\left[{ }^{\circ} \mathrm{C}\right]$ & -55 & -12 & -30 \\
\hline
\end{tabular}

Comparing of the three rubber materials, we can find that:

(1) The density of FKM is too high, so it will increase the weight of BPS;

(2) The tensile strength of HNBR is the best;

(3) The distortion resistances of AEM is the best;

(4) The compression resilience of FKM is the worst;

(5) The high temperature resistance of FKM is the best while its low temperature resistance is the worst.

Based on the analysis of the above, and also in consideration of the high cost of HNBR and FKM, this article chose AEM as the rubber material of BPS.

Skeleton material analysis. The commonly used material of skeleton is low alloy steel, such as HC420LA and ST12. The thickness of skeleton can be $1.3 \mathrm{~mm}, 1.5 \mathrm{~mm}, 2.0 \mathrm{~mm}$ or $3.0 \mathrm{~mm}$. Skeleton has a high demand on structural strength, dimension, flatness and burr. Therefore, the selection of skeleton is a techniqual difficulty, particularly the processing technology of skeleton.

\section{Structural design}

Generally, the structural design of BPS involves many factors. For instance, the thickness of the rubber material and the skeleton, the compression deformation, rigidity, and wear compensation of the lip, the strength and rigidity of BPS, the saving of materials, and the processing complexity of the structure all need precise calculation and design. In addition, as there is difference in the internal structure and shape of different gear shifting clutches in automatic transmission, the BPS used in each automatic transmission is different. And the BPS should be designed according to different clutch. Another difficulty in structural design is that sufficient space is needed for the arrangement of 
diaphragm spring. As a result, standardized production is not suited for BPS and accordingly the design difficulty and design costs are increased.

Take design of the BPS in B1 clutch of an in-development two-speeds automatic transmission for example. The given parameters are: The outside diameter of B1 clutch is $146.4 \mathrm{~mm}$, the outside diameter of hydraulic cylinder is $120 \mathrm{~mm}$, the inner diameter of cylinder is $50 \mathrm{~mm}$, the oil pressure is $1.2 \mathrm{MPa}$, the piston stroke is $4 \mathrm{~mm}$. According to the parameters, the compression of the BPS is designed to be $0.5 \mathrm{~mm}$, and the thickness of the rubber is designed to be $1 \mathrm{~mm}$, the thickness of the skeleton is designed to be $2 \mathrm{~mm}$, the overall dimension of the BPS is $49.6 \mathrm{~mm} \times 121 \mathrm{~mm} \times 19 \mathrm{~mm}$. And the structural drawing and partial enlarged drawing are shown in Fig. 2.
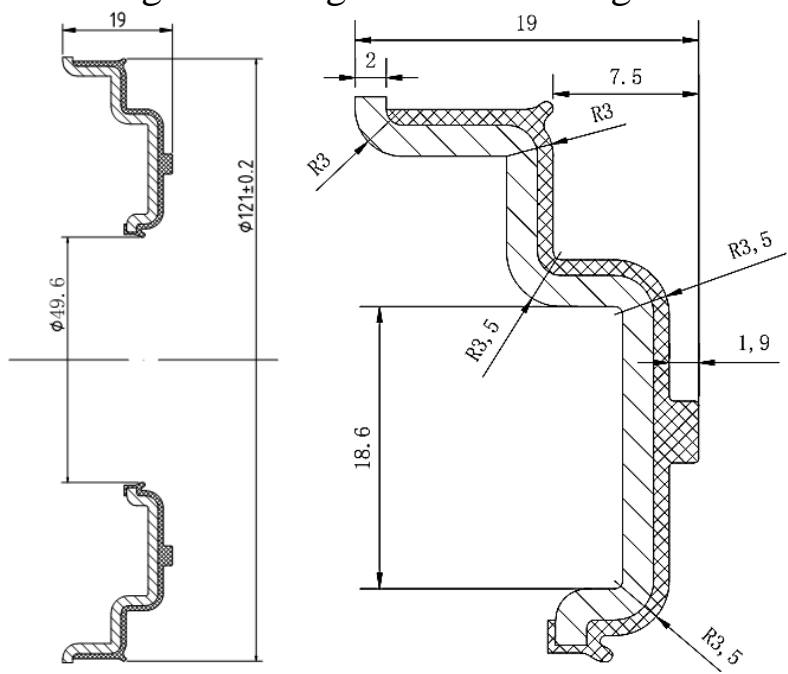

Fig. 2 The structural drawing and partial enlarged drawing of BPS

\section{Ansys analysis}

Finite element model. There are many difficulties in the research of the sealing performance of BPS. In the process of engineering design, designers design BPS mainly based on traditional experience. Before the experiment, the sealing performance of BPS is unpredictable, thus this method is inefficient. With the continuous development of finite element technique, software such as Ansys can be used to analyze the sealing performance of BPS. The main factor of failure of BPS is the deformation of the lip[3,4]. So Ansys is used to build models and analyze the pressure distribution of the upper lip and the lower lip of the BPS.

Approximately incompressible Mooney-Rivlin model is widely used as rubber material model, Eq. 1 can be used to represent Mooney-Rivlin model.

$$
W=C_{10}\left(I_{1}-3\right)+C_{01}\left(I_{2}-3\right)
$$

Where $\mathrm{C}_{10}$ and $\mathrm{C}_{01}$ are Mooney-Rivlin parameter. They can be calculated or obtained through experiments. $C_{10}$ and $C_{01}$ used in this article are 1.87 and $0.47[5]$.

When finite element model of the BPS is setting up, the inner wall of the cylinder and the skeleton are considered as a rigid body. That's because the elastic modulus of steel is far more than the elastic modulus of rubber materials. At the same time, there are some other assumptions:

(1)The rubber part is equivalent to a continuous, isomorphous and elastic material, and it has a certain Elastic modulus E and poisson's ratio v;

(2)The rubber part is approximately incompressible;

(3)The change of temperature is ignored.

Because the BPS is axisymmetric, the load of it is axisymmetric in an ideal case. Thus the three-dimensional model can be simplified to a two-dimensional one. Considered that the failure mainly occurs in the contact surface of BPS and cylinder, the model is built mainly to study the contact of BPS 
and cylinder. Thus the simplified model can simulate the contact of BPS and cylinder well, see Fig. 3. The stress distribution of the lips can be achieved through the finite element simulation, providing theoretical basis for the structural optimization of the lips.

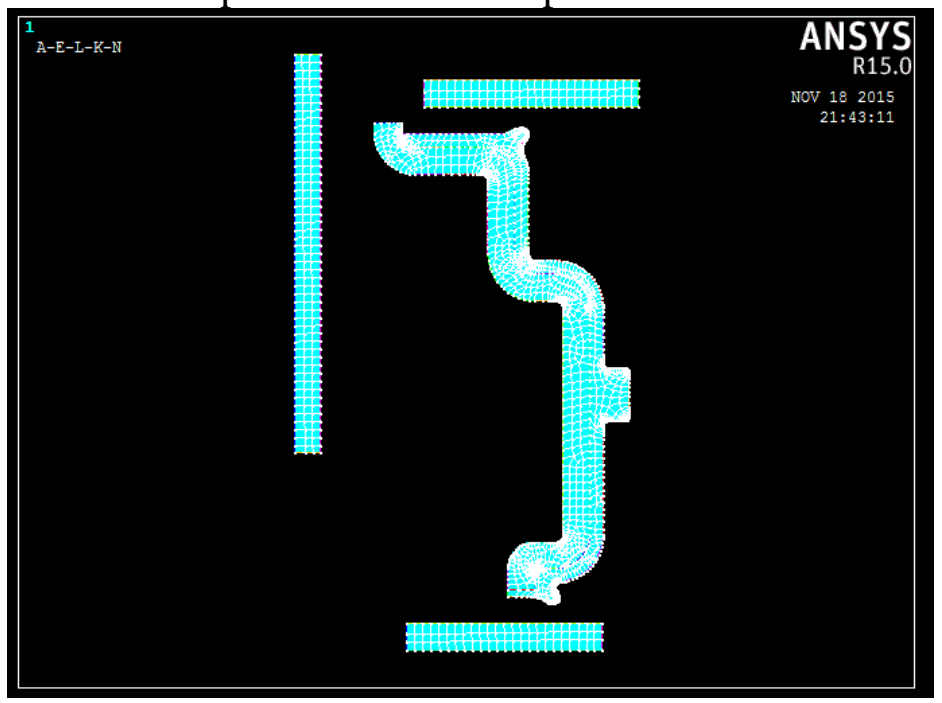

Fig. 3 Finite element model of the BPS

Constraint condition. Rubber unit chooses HYPER56, E=12.6Mpa, v=0.49; Skeleton unit and cylinder unit use PLANE42, E=2E5MPa, v=0.3. "Glue" command is used to connect rubber part and skeleton. Constraints are as follows:

(1) Put an X:-4mm and Y:0mm displacement for the BPS, to simulate the stroke;

(2) Put an X:0mm and Y:-2mm displacement for the upper cylinder, put an X:0mm and Y:1.8mm displacement for the lower cylinder, to simulate the assembly process;

(3) Put an equivalent oil pressure of 1.07Mpa which already gets rid of the spring force.

Ansys analysis. Consider that the shear strength of AEM is $5.7 \mathrm{MPa}$, thus the max contact stress must be less than 5.7MPa so that the BPS will not lose efficacy. What's more, the max contact stress must be larger than the oil pressure to ensure a normal sealing performance. In general, the max contact stress should be between 1.2MPa to 5.7MPa.

Structural optimization. The first time I got the calculation results of Ansys, I found that the max contact stress of the upper lip was $9.3 \mathrm{MPa}$, while that of the lower lip was $8.5 \mathrm{MPa}$. Both of them were not qualified. After that, I did lots of job to optimize the structure of the lip repeatedly (mainly increased curvature radius of some part of the lips), until the max contact stress was qualified. The optimization process is shown in Fig. 4.

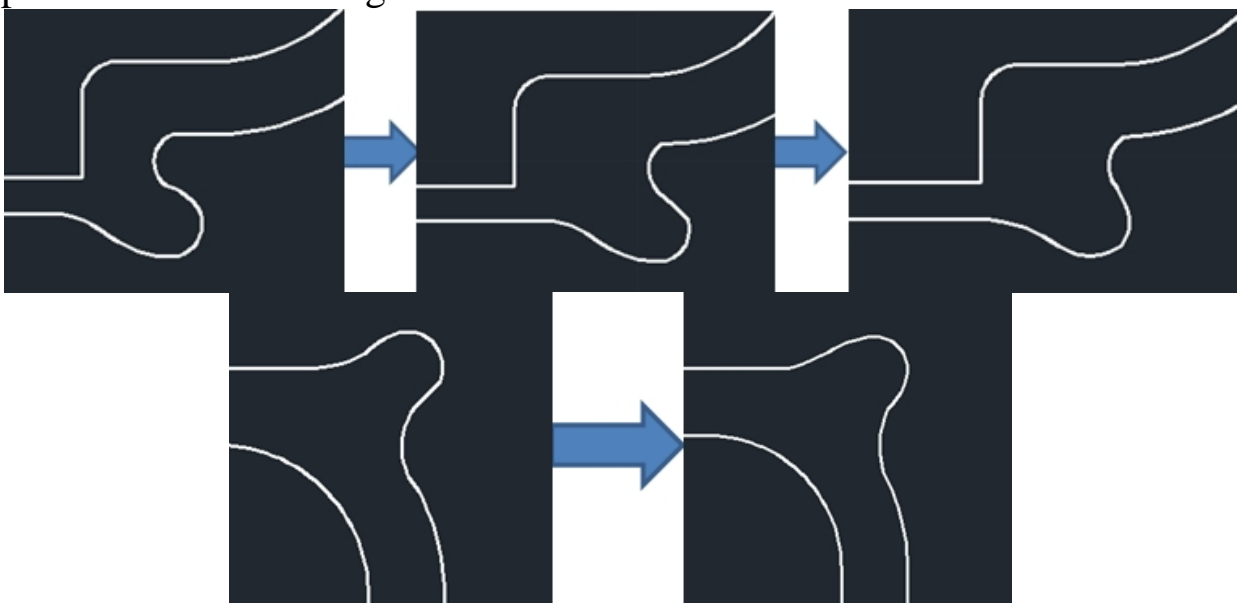

Fig. 4 The optimization process of the upper and lower lips

When the working conditions are set as $1.2 \mathrm{MPa}$ oil pressure and $4 \mathrm{~mm}$ stroke, the max contact stress of the optimized lower lip is $5.3 \mathrm{MPa}$, and the max contact stress of the optimized upper lip is $4.4 \mathrm{MPa}$. The contact stress distribution figure of the optimized BPS is shown as Fig. 5. 


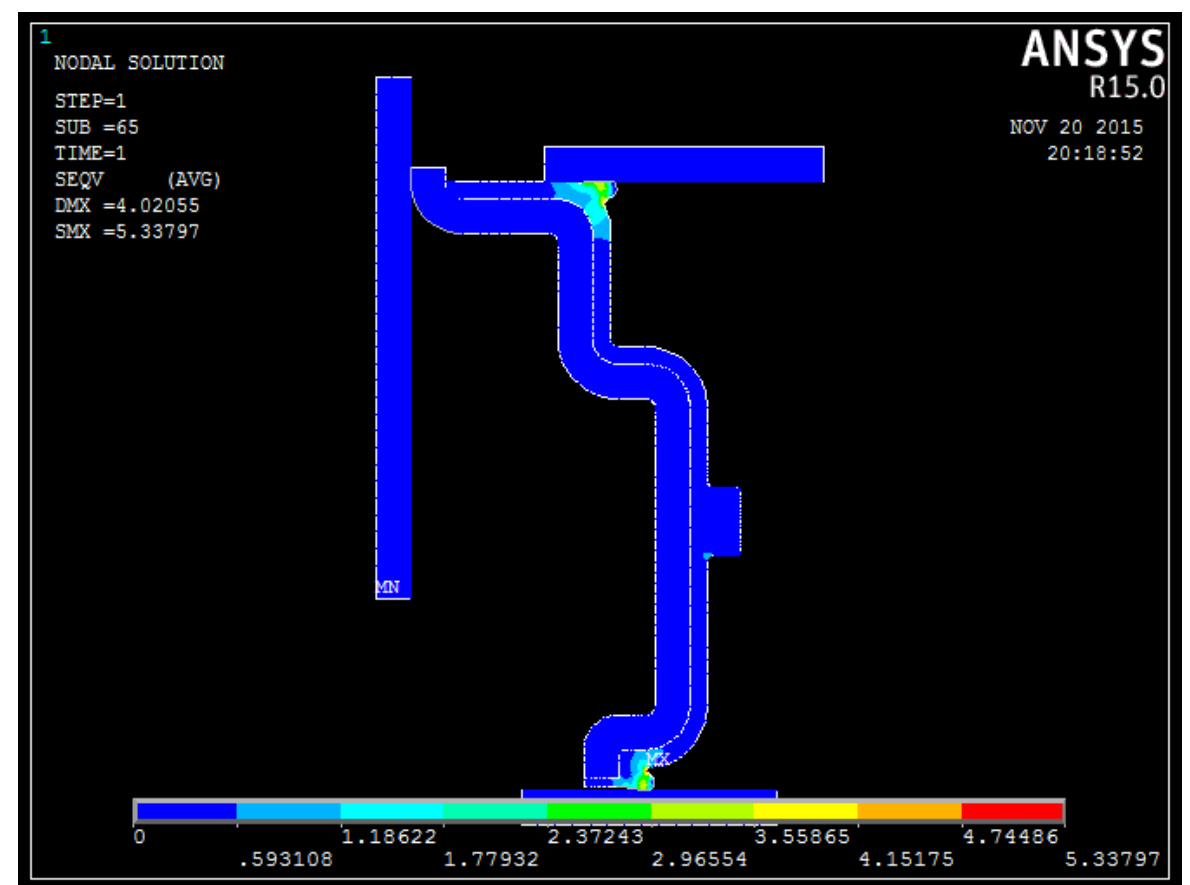

Fig. 5 The contact stress distribution figure of the optimized BPS

The simulation results of different working conditions are shown in Table 2.

Table 2 The simulation results of different working conditions

\begin{tabular}{l|c|l|c|c}
\hline \multirow{2}{*}{ Working condition } & \multicolumn{2}{|c|}{ Upper lip } & \multicolumn{2}{c}{ Lower lip } \\
\cline { 2 - 5 } & $\begin{array}{l}\text { Max contact stress } \\
{[\mathrm{MPa}]}\end{array}$ & $\begin{array}{l}\text { Contact } \\
\text { width }[\mathrm{mm}]\end{array}$ & $\begin{array}{l}\text { Max contact stress } \\
{[\mathrm{MPa}]}\end{array}$ & $\begin{array}{l}\text { Contact } \\
\text { width [mm] }\end{array}$ \\
\hline Assembly & 4.1 & 0.8 & 4.6 & 1.1 \\
\hline $\begin{array}{l}1.2 \mathrm{MPa} \text { oil pressure } \\
\text { and 4mm stroke }\end{array}$ & 4.4 & 0.9 & 5.3 & 1.1 \\
\hline
\end{tabular}

Creo is used to make 3 D structural drawings of the optimized BPS. See Fig. 6.
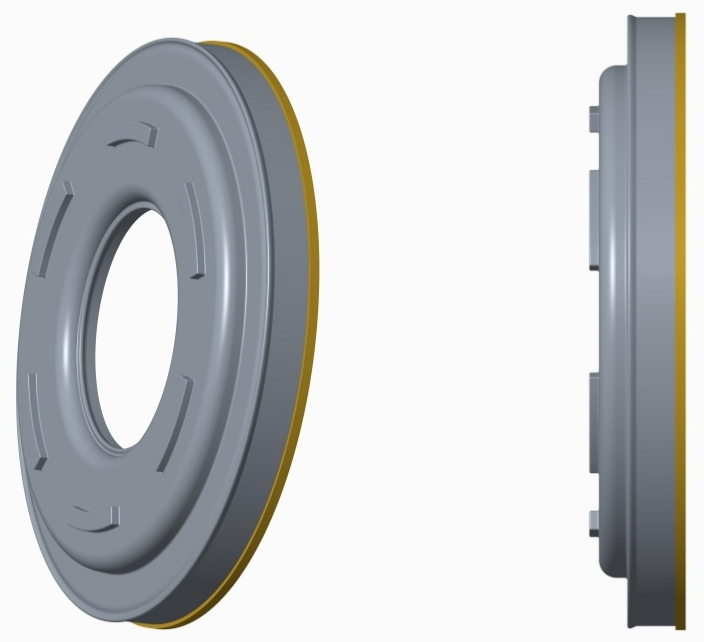

Fig. 6 Structural drawings of the optimized BPS

\section{Summary}

This article makes a detailed introduction to BPS technology and carries out a comprehensive analysis. After that, this article analyzes the working environment and the technical requirements of BPS, and also studies on rubber material characteristics of BPS. Then a design process of the BPS in a 
two-speeds automatic transmission of an electric vehicle is demonstrated. According to the design, Ansys is used to build models and analyze the contact stress distribution of the upper lip and the lower lip of the BPS. Then the sealing performance is analyzed to make structural optimization. Finally the simulation results of different working conditions and the structural drawings are displayed.

It is worth mentioning that the Bonded Piston Seal technology has just started in China. Thus the research contents of this article contribute to the industrial development of BPS. What's more, as the design object is BPS in automatic transmission of electric vehicle and electric vehicle is a hot topic, so I hope it has a certain value.

\section{Acknowledgements}

This work was supported by the Fundamental Research Funds for the Central Universities, and was also supported by the Beijing Key Laboratory for High-efficient Power Transmission and System Control of New Energy Resource Vehicle.

\section{References}

[1] Komiya, E., Yomogida, M., Kanzaki, Y., Nishina, H., Development of bonded piston seals, SAE 2001 World Congress.

[2] V. E. Shcherba, et al. Analysis of Compressor-Pump Piston Seal Formed from Concentric Slit with Isolated Channel in Piston Body, Chemical and Petroleum Engineering, 2014, 501-2.

[3] Hirano F, Kaneta M, Dynamic behavior of flexible seals for reciporocating motion, Proc of the 4th Int Conf on Fluid Sealing, (1969) pp. 11-20.

[4] T. G. Roust. An Experimental and Theoretical Study of Pressure and Thermal Distortions in a Mechanical Seal[J], ASLE Transactions, 1986, 29 (2): 151-159.

[5] XU Tong-jiang, Finite element analysis of O ring based on Ansys, 2012. 